\title{
Exaggerated Placental Site Reaction Following a Term Pregnancy: A Case Report
}

\author{
Rajeev Sen ${ }^{1}$, Bharti Sharma ${ }^{2}$, Ritesh Kumar Sheorain ${ }^{2}$, Nikita Prasad ${ }^{3 *}$, Pushpa Bisht ${ }^{3}$, Swarnim Dalakoti ${ }^{3}$ \\ ${ }^{1}$ Senior Professor and Head, ${ }^{2}$ Senior Resident, Department of Pathology, ${ }^{3}$ Junior Resident, Department of Pathology, Pandit Bhagwat Dayal Sharma \\ Post Graduate Institute of Medical Sciences (PGIMS), Rohtak, Haryana 124001, India
}

DOI: $\underline{10.36348 / \text { sjpm.2020.v05i08.006 }}$

| Received: 04.08.2020 | Accepted: 17.08.2020 | Published: 20.08.2020

*Corresponding author: Nikita Prasad

\section{Abstract}

Exaggerated placental site (EPS) reaction is an exuberant physiologic process wherein intermediate trophoblasts infiltrate the underlying endometrium and myometrium at the implantation site. Its diagnosis is established only histopathologically which reveals proliferation of intermediate trophoblasts at the implantation site with absence of mitotic activity. Distinguishing EPS reaction from other trophoblastic tumors is crucial due to difference in management and prognostic outcome. We reported a case of 35 years old multigravida female who presented to the hospital at gestational age of $39^{+6}$ weeks with complaints of persistent post-delivery bleeding. We received a hysterectomy specimen in our department. Grossly the specimen measured $14 \mathrm{~cm}$ x $14 \mathrm{~cm} \times 8 \mathrm{~cm}$ and endometrial cavity was filled with hemorrhagic soft tissue. On microscopy, microsections revealed gravid uterus with numerous intermediate trophoblasts extending into but confined to the myometrium; a diagnosis of exaggerated placental site rophoblastic reaction was made. EPS can develop anytime from early to term pregnancy. Clinical features are non-specific such as abdominal pain, high grade fever, continuous bleeding per vaginum. Only 12 cases have been reported in English literature based on PubMed from 1990 through 2020. EPS is rare and diagnosis is chiefly histopathological. It should be considered albeit rare as a differential diagnosis of heavy bleeding after a delivery or abortion. It is necessary to differentiate this entity from other neoplastic pathologies of trophoblast, since it does not require any surgical treatment or follow up.

Keywords: Exaggerated placental site, intermediate trophoblast, Myometrium.

Copyright @ 2020: This is an open-access article distributed under the terms of the Creative Commons Attribution license which permits unrestricted use, distribution, and reproduction in any medium for non-commercial use (NonCommercial, or CC-BY-NC) provided the original author and sources are credited.

\section{INTRODUCTION}

Exaggerated placental site (EPS) reaction is an exuberant physiologic process wherein intermediate trophoblasts infiltrate the underlying endometrium and myometrium at the implantation site. It can occur following normal pregnancy, ectopic pregnancy, molar pregnancy or an abortion [1].

Its diagnosis is established only histopathologically which reveals proliferation of intermediate trophoblasts at the implantation site with absence of mitotic activity. Distinguishing EPS reaction from other trophoblastic tumours is crucial due to differences in management and prognostic outcome. It is imperative to consider this pathology and take biopsies from suspicious lesions within the placental site for pathologic examination [2].

\section{CASE REPORT}

We reported a case of 35 years old multigravida female $\left(\mathrm{G}_{3} \mathrm{P}_{1} \mathrm{~L}_{1} \mathrm{~A}_{1}\right)$, with a previous history of LSCS who presented to the hospital at gestational age of $39^{+6}$ weeks, with complaints of bleeding per vaginum and mild pallor. An emergency caesarean section was done under GA followed by peripartum hysterectomy and bladder repair. This was in view of persistent post-delivery bleeding, for which conservative management failed and disseminated intravascular coagulation (DIC) ensued. The hysterectomy specimen was sent for histopathological examination. Grossly the specimen measured $14 \mathrm{~cm} \mathrm{x}$ $14 \mathrm{~cm} \times 8 \mathrm{~cm}$, endometrium measures $0.5 \mathrm{~cm}$ and myometrium measures $3.5 \mathrm{~cm}$ (Figure 1, Figure 2). The endometrial cavity was found to be filled with haemorrhagic soft tissue pieces. Microsections revealed gravid uterus with numerous intermediate trophoblasts, showing abundant eosinophilic cytoplasm and hyperchromatic irregular nuclei. These intermediate trophoblasts were seen to be extending into, but confined to, the myometrium (Figure 3, Figure 4). Based on the histopathological features, a diagnosis of exaggerated placental site trophoblastic reaction was made. 


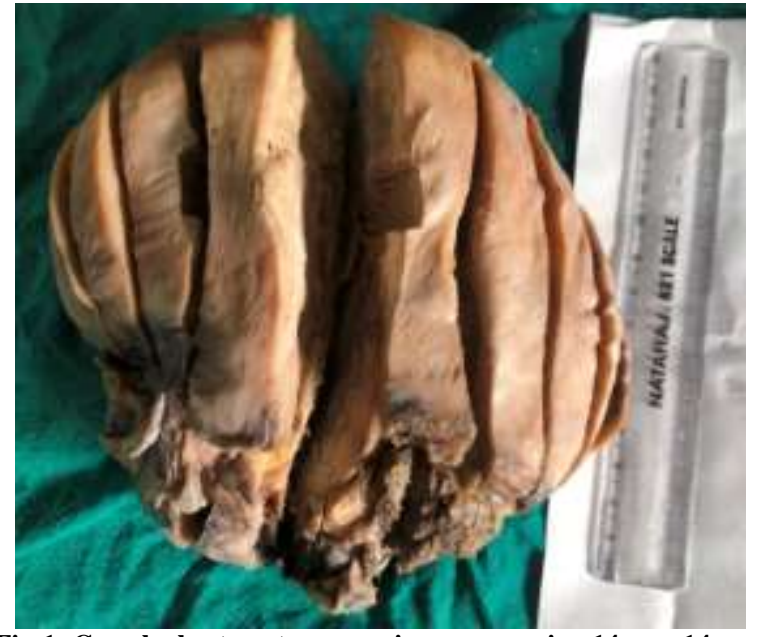

Fig-1: Grossly, hysterectomy specimen measuring $14 \mathrm{~cm}$ x $14 \mathrm{~cm} x$ $8 \mathrm{~cm}$.

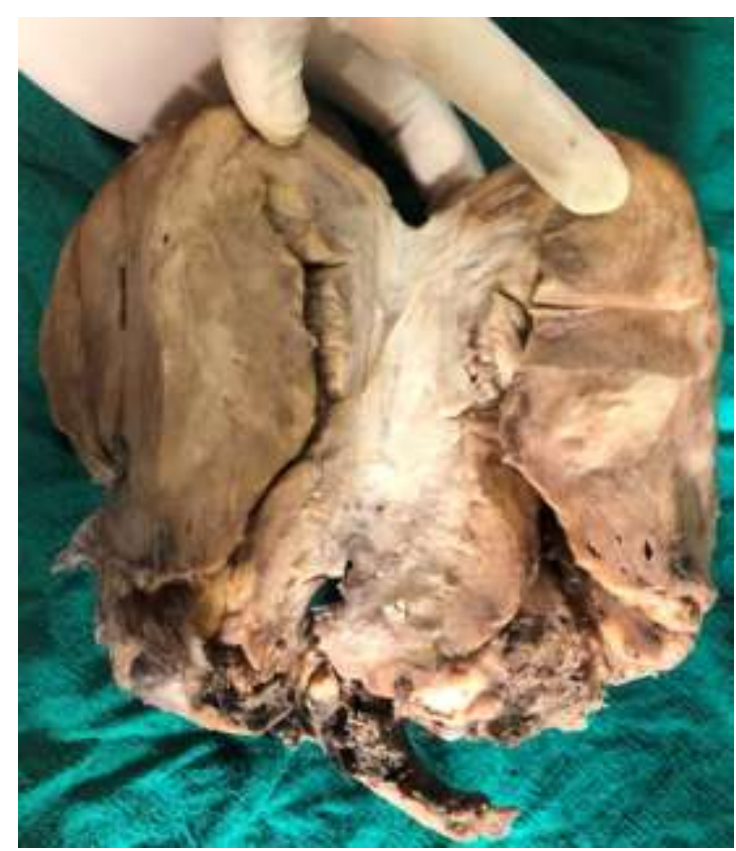

Fig-2: Gross examination showing serial sectioning of hysterectomy specimen; endometrium measuring $0.5 \mathrm{~cm}$ and myometrium measuring $3.5 \mathrm{~cm}$

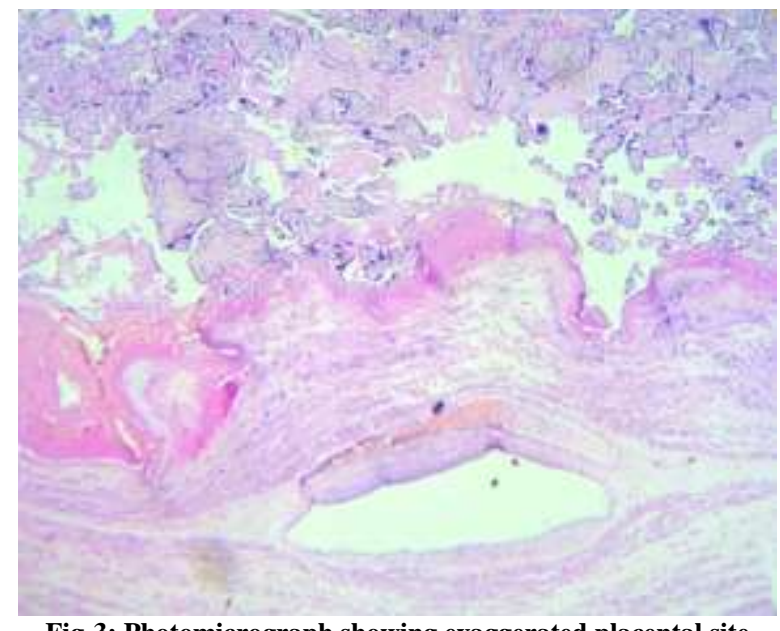

Fig-3: Photomicrograph showing exaggerated placental site reaction. $(\mathrm{H} \& \mathrm{E} ; \mathbf{1 0 0 X})$

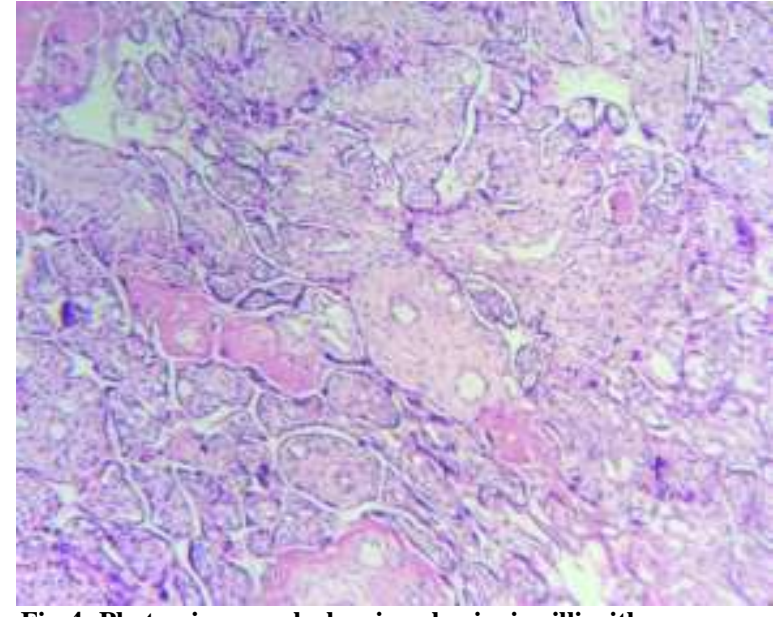

Fig-4: Photomicrograph showing chorionic villi with numerous intermediate trophoblasts $(\mathrm{H} \& \mathrm{E} ; \mathbf{4 0 0 X})$

\section{DISCUSSION}

EPS reaction represents part of the spectrum of normal implantation site change. It represents an exaggeration of the normal reaction at the placental site, with proliferation of intermediate trophoblasts in both endometrium and superficial myometrium, with or without deep invasion of the myometrium. EPS can develop anytime from early to term pregnancy. It has been detected in association with molar pregnancy, cervical pregnancy, abortion or induced abortion of early pregnancy, intrauterine fetal death of 24 weeks gestation and term pregnancy [3].

Clinical features are non-specific such as abdominal pain, high grade fever, continuous bleeding per vaginum, which may or may not be associated with a mass lesion or atonicity of the uterus. A review article demonstrated that most cases of EPS appear in the form of a mass, although it is defined as the extreme end of a physiological process rather than a true lesion [3]. This led to the premise that EPS may be a precursor lesion of PSTT [4].

Histopathologically, the lesion consists of an excessive infiltration of implantation site with intermediate trophoblasts (ISIT), along with multinucleate giant cells. The overall endomyometrial anatomy remains unaltered. There may be some nuclear atypia, but mitotic figures are characteristically absent [5].

The distinction between a normal placental site and EPS is somewhat arbitrary due to lack of reliable data quantifying the extent of trophoblastic infiltration at different stages of normal gestation [6]. The pathological significance of EPS has not been clearly determined. It cannot be diagnosed clinically, and has not received much attention until now. In fact, only twelve cases have been reported in English literature, based on PubMed from 1990 through 2020. 
Differential diagnoses include entities with proliferation of the intermediate trophoblast, such as placental site nodule, placental site plaque and placental site trophoblastic tumor [1]. Lack of a mass lesion and low ki-67 labelling index $(<1 \%)$ distinguish EPS from placental site trophoblastic tumours. EPS associated with molar pregnancy may show greater atypia and higher ki-67 index of 5-10\% [7]. The immunohistochemical markers are confirmatory, although not mandatory, including ki-67, cytokeratin 18 and p63. The ki-67 labeling index is $0-1 \%$ in EPS as compared to $>10 \%$ in placental site trophoblastic tumour. Chorionic villi are usually present and unremarkable.

ISITs infiltrate the decidua and myometrium, and a portion of them replace the arterial walls of the implantation site to establish maternal-fetal circulation. The intermediate trophoblasts function as an anchor of placenta to the maternal tissue in order to maintain pregnancy, but their role post-partum is unclear [1]. It has been speculated that their excessive proliferation can cause atonicity of the uterus, resulting in severe postpartum haemorrhage. Further studies are needed to support this hypothesis. If an association between atonicity of uterus and excessive infiltration of ISITs, or EPS is proved, it may contribute to the development of a diagnostic method to predict development of atonic uterus.

Most cases in literature were reported on hysterectomy specimens, performed in view of controlling severe uterine haemorrhage or preventing development of gestational trophoblastic disease. The present case was also diagnosed on histopathological examination of hysterectomy specimen of a term pregnancy.

\section{CONCLUSION}

EPS has morphological and immunohistochemical features of normal intermediate trophoblast at the implantation site. It is rare and diagnosis is chiefly histopathological. It should be considered, though rare, as a differential diagnosis of heavy bleeding after a delivery or an abortion [1]. Pathological examination should be performed to evaluate the status of ISITs infiltration when the uterus is resected in the postpartum period. It is necessary to differentiate this entity from other neoplastic pathologies of trophoblast, since it does not require any specific treatment or follow-up.

\section{REFERENCES}

1. Jayakrishnan, N., Jayakrishnan, K. (2016). A placental dilemma: exaggerated placental site tumour. Int J Reprod Contracept Obstet Gynecol, 5, 2425-7.

2. Akbayir, O., Alkis, I., Corbacioglu, A., Ekiz, A., Akca, A., \& Cekic, S. (2012). Exaggerated placental site reaction detected during caesarean delivery: a case report. Clinical and Experimental Obstetrics and Gynaecology, 39(2), 234.

3. Takebayashi, A., Kimura, F., Yamanaka, A., Takahashi, A., Tsuji, S., Ono, T., \& Murakami, T. (2014). Exaggerated placental site, consisting of implantation site intermediate trophoblasts, causes massive postpartum uterine hemorrhage: case report and literature review. The Tohoku Journal of Experimental Medicine, 234(1), 77-82.

4. Liu, G., Yuan, B., \& Wang, Y. (2013). Exaggerated placental site leading to postpartum hemorrhage: a case report. The Journal of Reproductive Medicine, 58(9-10), 448-450.

5. Young, R. H., Kurman, R. J., \& Scully, R. E. (1988, May). Proliferations and tumors of intermediate trophoblast of the placental site. In Seminars in diagnostic pathology, 5(2), 223 237.

6. Shih, I. M., \& Kurman, R. J. (2001). The pathology of intermediate trophoblastic tumors and tumorlike lesions. International journal of gynecological pathology, 20(1), 31-47.

7. Murdock, T. A., Veras, E. F., Kurman, R. J., \& Mazur, M. T. (2019). Gestational Trophoblastic Disease. In Diagnosis of Endometrial Biopsies and Curettings (pp. 75-119). Springer, Cham. 